\title{
Integrative air quality management airshed by using multigas passive sampling technology in Canada
}

\author{
H. Tang \& F. Apon \\ AMEC Environmental and Infrastructure, Edmonton, Alberta, Canada
}

\begin{abstract}
Environmental impacts including air pollution have become more important as the world toward sustainable development. To properly assess a large area's impact of air pollution requires modeling prediction and/or real measurement by using active and/or passive methods. Real-time active methods can provide instant measurement results, which is an advantage that passive technology does not have. But passive technology is easy to use and very cost effective. Therefore, many air pollution monitoring networks normally combine active and passive methods together. In the past decades, a new strategy for air quality management has been developed in Canada. It is an integrative air quality management or airshed management zone (AMZ), which includes local stakeholders and therefore can extensively use local resources to address many local concerns. The application of a newly patented technology - Multigas Passive Sampling Technology in the AMZ will be described.

Keywords: environmental impact, air pollution, passive sampler, air monitoring.
\end{abstract}

\section{Introduction}

Environmental impacts are affecting sustainable development, and as such have become increasingly influential in the policies of national governments and nongovernmental organizations. The Environmental Impact Assessment (EIA) is a concept and method developed many years ago in the environmental policy arena to evaluate potential consequences of human activity on the natural environment. Therefore, EIA is a tool for sustainable development. It entails identifying and evaluating both intended and unintended consequences and outcomes associated with a particular intervention. Air pollution assessment is 
one of the important parts in the EIA. There are normally two technologies used for air pollution assessment: model prediction and real measurement. This paper will concentrate on real measurement.

It is well known that there is almost no clear boundary to define an environmental impact area. Therefore, the trans-boundary impacts always occur. Thus stakeholder involvement becomes a necessary component for both successful EIA systems and EIA studies. In order to address a regional issues and increase different regional cooperation under trans-boundary impacts, a new integrative air quality management system (IAQM) or airshed management zone (AMZ) has been developed and organized in Canada since 1995 [1].

\section{Integrative air quality management system}

Many air quality issues are local, both in their cause and the solutions required. In these cases, province-wide approaches for air quality management may not be appropriate or efficient [2]. Also local individual measurements (such as a chemical plant) can only concentrate its own air quality; the effects for other places cannot be addressed. But a local air quality management zone can provide an opportunity for local stakeholders to design local solutions to their own concerns in an integrative and economic way. Consequently many individual air monitoring stations can be eliminated.

In recent years, a new strategy for air quality management has been developed in Canada. This management strategy can be named as integrative air quality management system (IAQM) or Airshed Management Zone (AMZ). The AMZ combines all air pollution measurement technologies and deploys them in a manner that provides a reasonable expression of the air quality in a region.

An AMZ includes multi-stakeholders in the region which are from provincial and local governments, industry, transportation, agriculture, local communities, and local environmental groups. The primary responsibility of the AMZ is to develop a management plan to deal with air quality concerns in the region and cooperate with other AMZ dealing with some problems such as trans-boundary impacts. The issues are defined broadly, reflecting the vision and principles of air quality management. The success of an AMZ is largely dependent on the cooperation and dedication of all stakeholders.

The aim of a regional $\mathrm{AMZ}$ is to monitor air quality and its impact on local sustainable development and human health issues, and seek remedies for the air quality problems within the region. Some other potential impacts of air quality on special issues such as soil acidity, crops and forests can also be identified in the zone. Bio-monitoring research projects will be used to collect data for a number of years; then results can be fully analyzed.

Normally, an AMZ is a non-profit society established under the local society act of Alberta Canada. The AMZ board of directors who represent different sectors is volunteer worker. So far, there are already 9 AMZ existing in Alberta Canada (Figure 1). More AMZ will be established in the future. 


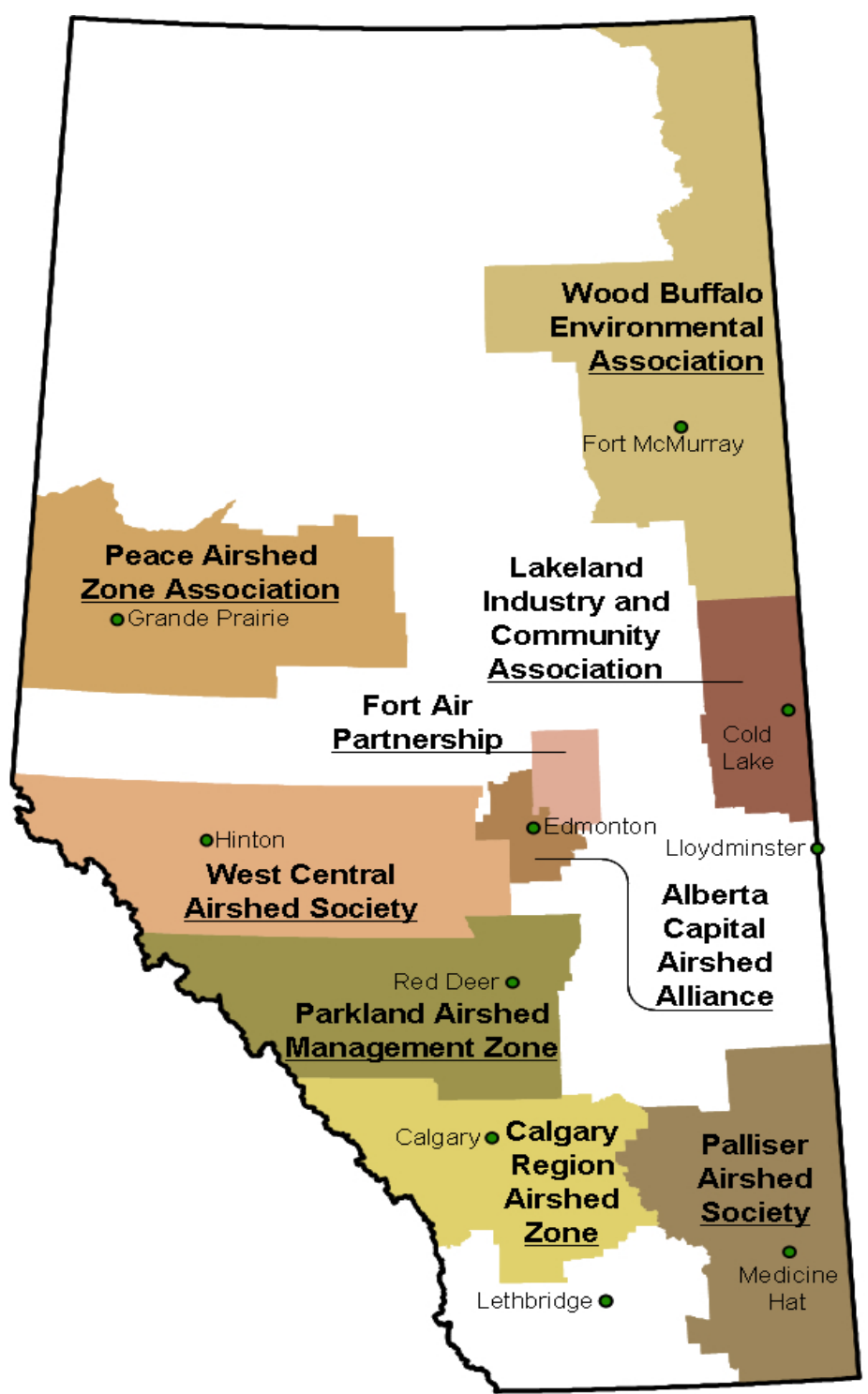

Figure 1: $\quad$ Existing airsheds in Alberta Canada.

$\mathrm{AMZ}$ are funded by major industries operating in the region. The amounts paid are proportional to their pollution released to the local air. Other member contributions to the fund are in kind as well as monetary.

Normally there are several purposes to establish an AMZ such as providing a forum for discussion and co-ordination of matters related to an airshed monitoring and management program for the zone; promoting understanding between the society, governments, stakeholders, the public, other airshed zones 
and other organizations about environmental impacts of activities inside and outside the airshed; identifying and quantifying on a credible basis air quality concerns, and using the data to recommend and implement airshed management solutions for the AMZ; coordinating and integrating airshed monitoring and management activities within the AMZ with the provincial policies and guidelines.

\subsection{Parkland airshed management zone - an example}

The Parkland airshed management zone (PAMZ) is one of the 9 AMZ in Alberta of Canada [3]. The fundamental principle of the PAMZ is to provide communications among stakeholders.

The PAMZ has been operating for 15 years since established in 1997 following the Clean Air Strategic Alliance (CASA) model of consensus decision making in Alberta Canada [1]. Its boundaries cover a 45,000 square kilometers in the west central Alberta (Figure 1). The population living in this area is about 220,000 . Oil and gas processing, agriculture, petrochemical, forestry and tourism are major industries in the PAMZ. The PAMZ has a very diverse natural region including Rocky Mountains in the west and the grassland in the east.

\subsubsection{Data collection}

Active real-time instruments (continuous air pollution analyzers) and passive sampling technology are used in the PAMZ. The air pollution analyzers can monitor $\mathrm{SO}_{2}, \mathrm{H}_{2} \mathrm{~S}, \mathrm{O}_{3}, \mathrm{NO}_{x}, \mathrm{CO}, \mathrm{PM} 10$ etc. Thirty passive sampling stations are equipped with the All Season Passive Sampler (ASPS) developed by Tang et al. before 2002 [4-7]. Figure 2 shows the active and passive station locations in the PAMZ, where the brawn squares are for passive stations and the green triangles are for continuous air monitoring stations. Figure 3 is one of the continuous air monitoring stations and Figure 4 is a picture of a passive station in the PAMZ.

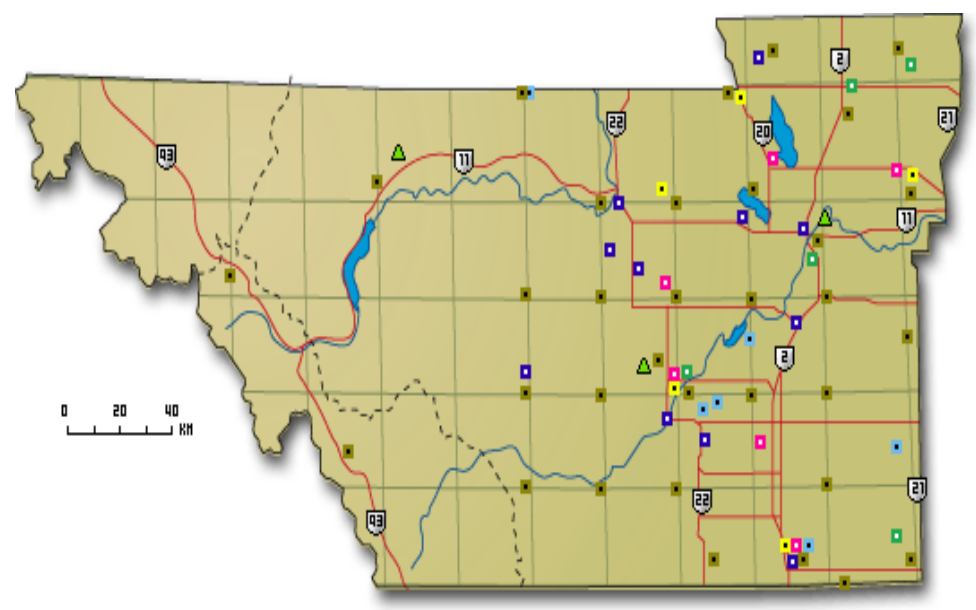

Figure 2: $\quad$ Air monitoring locations in the PAMZ. 


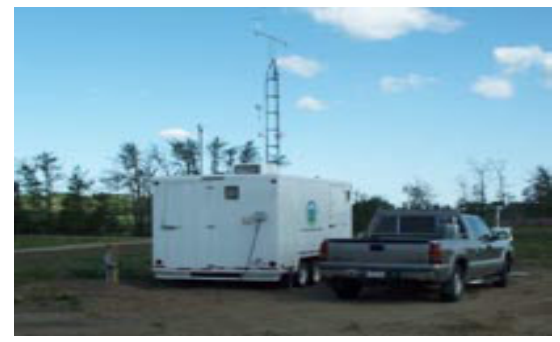

Figure 3: $\quad$ PAMZ continuous station.

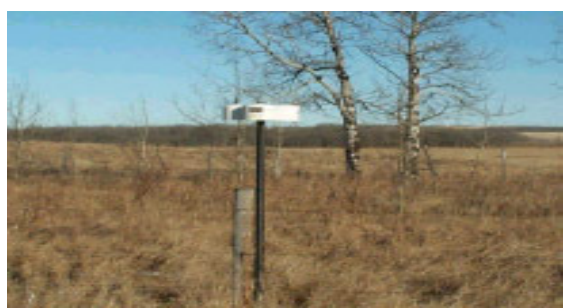

Figure 4: A field passive sampler station in PAMZ.

The continuous stations in the PAMZ can provide instant information through the website; but the data are not corrected by a QA/QC process. The QA/QC checked data for the continuous and passive samplers in the PAMZ are available through the CASA Data Warehouse [1].

Figure 5 shows a $\mathrm{SO}_{2}$ concentration distribution trend in the PAMZ measured by the ASPS passive sampler. It clearly shows that the west Rocky Mountain area has less $\mathrm{SO}_{2}$ than the east grassland area. The PAMZ data collections clearly demonstrate that the use of passive sampling technology over much of the AMZ provides cost-effective means.

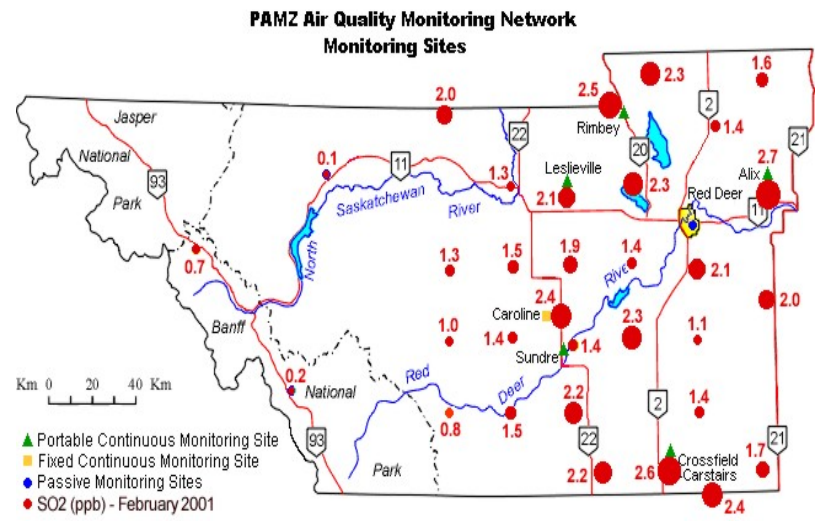

Figure 5: $\quad$ A monthly $\mathrm{SO}_{2}$ contribution trend in PAMZ. 


\section{AMEC multigas passive sampling system - a patented technology}

\subsection{Passive sampling technology}

Over the past few decades, many sampling methods for monitoring air pollutants in ambient and indoor air have been developed and subsequently improved. These methods can generally be classified as active methods and passive methods.

Active methods directly pump air through collection devices to collect air pollutants. Therefore electrical power, roads and shelters are required to operate the active real-time samplers. A passive (or diffusive) sampler is a device which is capable of taking samples of gas or vapor pollutants from the atmosphere at a rate controlled by a physical process such as diffusion through a static air layer or permeation through a membrane. However, it does not involve the active movement of the air through the sampler. Passive samplers are generally simple in structure and do not require electricity. Therefore, passive samplers, on the other hand, are cost effective and convenient to use.

The major difference between active samplers and passive samplers is that the active sampler has a known sampling rate, which is the pump's flow rate. The passive sampler's sampling rate depends on many factors such as temperature, relative humidity (RH), wind direction, wind speed, sampler's structure, collection media etc. If a passive sampler is to be used in all climate conditions, there must be a reasonable way to calculate the sampling rate. It would be highly unreasonable to expect that a passive sampler's sampling rate would not vary from $-30^{\circ} \mathrm{C}$ to $+30^{\circ} \mathrm{C}$ and from $90 \% \mathrm{RH}$ to $15 \% \mathrm{RH}$. Therefore, the key factor for using passive samplers is how to determine their sampling rates. Tang et al. have reported using equations to address the problems [4-7]. Field applications demonstrate that Tang's approach was practically useful in many cases.

\subsection{All-season passive sampling technology}

With the support of Alberta Environmental Protection (AEP), Alberta Research Council (ARC), Clean Air Strategic Alliance of Alberta (CASA) and the National Research Council of Canada (NRC), Tang et al. [4-7] successfully developed an all-season Passive Air Sampling System (ASPS) for sampling $\mathrm{SO}_{2}$, $\mathrm{NO}_{2}, \mathrm{O}_{3}$ and $\mathrm{H}_{2} \mathrm{~S}$ in the atmosphere from 1996 to 2003 (see Figure 6). The unique features of the ASPS are:

* A new passive sampler body and a rain shelter were designed allowing the passive sampler be installed face downwards outdoors. This design helps the ASPS avoid dust problems and facilitates air movement parallel to the diffusion barrier surface (Figures 6).

* An equation, which accounts for variations in temperature, relative humidity and wind speed, is used to calculate sampling rates. 
* The ASPS is very sensitive. It can be used to collect ppt levels of $\mathrm{H}_{2} \mathrm{~S}$, sub ppb levels of $\mathrm{SO}_{2}, \mathrm{NO}_{2}$ and $\mathrm{O}_{3}$ in air.

* The collection time is very flexible. It can be exposed from 4 hours up to six months depending on the monitored pollutant and its average concentration in air.

* By attaching a clip to the sampler body, it can be used as a personal monitor.

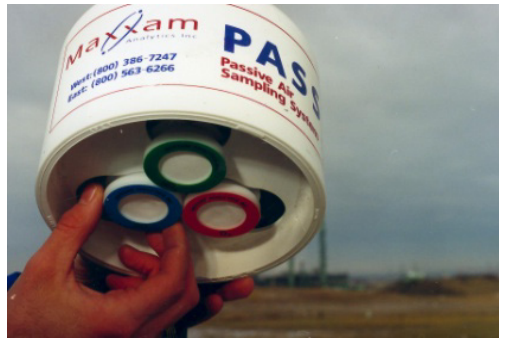

Figure 6: The all-weather passive air sampling system.

The downward facing installation of the passive samplers can prevent the precipitation of suspended particles in the atmosphere on the surface of the diffusion barriers. This prevents the air pollutants from being absorbed by the particles and avoids the pore-size change of the diffusion barrier, which will eventually change the sampling rate. Face downward installation also helps to obtain air movement across the diffusion barrier surface of a passive sampler.

Although Tang's above invention has been successfully used in many projects in the world, further studies revealed that some significant errors still occurred in different occasions [8]. Improvements of the ASPS are needed.

\subsection{The AMEC multigas passive sampling system}

In order to reduce cost and save our environment, several scientists have studied different ways to address the above problems. Tang et al. tested a collection medium which could be used to simultaneously sample $\mathrm{HF}, \mathrm{NO}_{2}$ and $\mathrm{SO}_{2}$ from 2008 to 2009 [9]. In the Ogawa passive sampler, due to two separate sampling chambers in each side, thus, it can be easily installed two different collection media. A study for sampling $\mathrm{NO}_{2}$ and $\mathrm{NO}_{\mathrm{x}}$ together has been reported [10].

A new generation of passive sampling technology - multigas passive sampling system (MGPS) has been reported [11, 12] and patented. Different from the normal passive sampler that use one collection medium for collecting a single or a group of air pollutants such as active charcoal for volatile organic compounds (VOC), the multigas passive sampling system (MGPS) (Figure 7) uses one passive sampler body to pack several different collection media at the same time to collect several air pollutants. In the MGPS, VOC or aldehyde is considered as one (group) air pollutant. 


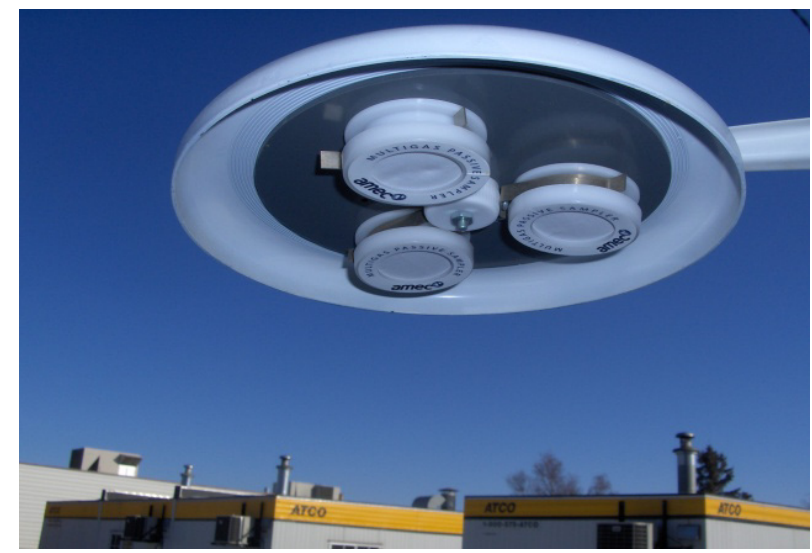

Figure 7: $\quad$ AMEC multigas passive sampling system.

The AMEC MGPS passive sampler body with a newly designed insert is shown in Figures 8. The MGPS can be used for both indoor, ambient air quality and personal exposure studies. The insert can be used to install 4 different collection media as the first layer in the passive sampler body. For more pollutants, a second layer or more layers can also be used. In this paper, we only report the first layer study.

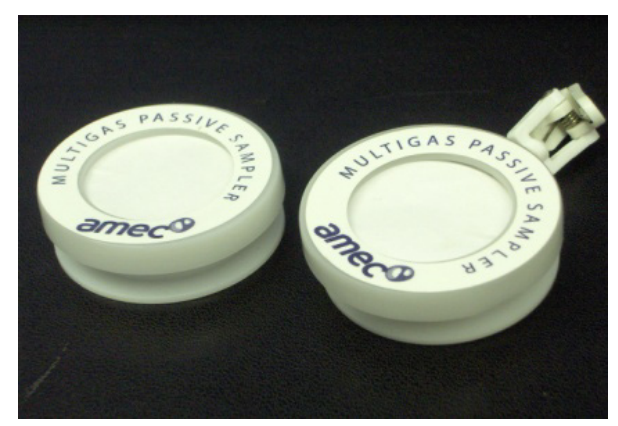

Figure 8: $\quad$ AMEC MGPS passive samplers for indoor, ambient and personal exposure uses.

The advantages of the MGPS include:

- $\quad$ Environment friendly, which can save materials, chemicals, wastes, etc.

- Cost effective.

- Convenient to use, which can save labor for field jobs and passive sampler management.

- Flexible for clients, in which one to several collection filters can be installed based on clients' need.

- $\quad$ More accurate. 


\subsection{Comparison of the AMEC multigas passive sampling system and the all-season passive sampling system}

The MGPS has two significant advantages compared to the ASPS: cost effective and more accurate. Table 1 lists comparisons of sampling 4 air pollutants in triplicate by using MGPS and ASPS. It can be seen that the MGPS save $75 \%$ compared to the ASPS.

Table 1: $\quad$ Four air pollutants collected in triplicate by ASPS and MGPS.

\begin{tabular}{|c|c|c|c|c|}
\hline ITEM & No. for ASPS & No. for MGPS & No. Saved & \% Saved \\
\hline Rain shelter & 4 & 1 & 3 & 75 \\
\hline Passive body & 12 & 3 & 9 & 75 \\
\hline Diffusion Barrier & 12 & 3 & 9 & 75 \\
\hline Collection filter & 12 & 3 & 9 & 75 \\
\hline Chemicals* & 12 & 3 & 9 & 75 \\
\hline Waste generated** & 12 & 3 & 9 & 75 \\
\hline Field installation & 12 & 3 & 9 & 75 \\
\hline Average & & & & 75 \\
\hline
\end{tabular}

*Comparison of chemicals implies total chemicals used for one collection medium as one unit. In this case, 12 units are used for ASPS; only 3 are used for MGPS.

** Comparison of waste generated uses extraction volume for one collection medium as one unit. In this case, 12 collection media are used by ASPS, which generate 12 unit wastes; but only 3units are generated in MGPS.

The ASPS sampling rate was calculated by an equation associated with temperature, relative humidity and wind speed published by Tang before (Equation 1).

$$
\mathrm{R}_{\mathrm{S}}=\mathrm{A} \mathrm{T}^{1 / 2}+\mathrm{BRH}+\mathrm{CWSP}-\mathrm{D}
$$

Where $R_{S}$ is the air pollutant sampling rate, $\mathrm{ml} / \mathrm{min}$; $\mathrm{T}$ is average temperature of the sampling period; $\mathrm{RH}$ is average humidity (\%); WSP is average wind speed, $\mathrm{cm} / \mathrm{sec}$, if $\mathrm{WSP}>130$, then $\mathrm{WSP}=130, \mathrm{~A}, \mathrm{~B}, \mathrm{C}$ and $\mathrm{D}$ are constants, but varying from pollutant to pollutant.

The MGPS sampling rate determinations are using a newly developed Integrative Data Management System (IDMS). Practices have demonstrated that the new IDMS can generate results with very low standard deviations.

It is well known that passive sampling rate depend on many factors. In addition to temperature, relative humidity, and wind speed, other factors such as atmospheric pressure, local terrain, chemicals in the atmosphere etc. also play important roles. Tang et al. [4-7] simplified the sampling rate calculation through only using temperature, relative humidity, and wind speed; it did provide 
a useful tool for accurately monitoring air quality in many cases; but in some other cases, it generated large deviations, noted previously.

Table 2 lists an inter comparison of relative errors generated by the MGPS and the ASPS in an Alberta study. It can be seen that the MGPS results are better than the ASPS. The average relative errors are $35 \%$ for ASPS and only $7 \%$ for MGPS.

Table 2: $\quad$ Relative errors inter comparison between MGPA and ASPS.

\begin{tabular}{|c|c|c|c|c|}
\hline Error\% & SO2 & O3 & NO2 & Average \\
\hline ASPS & 24 & 30 & 50 & 35 \\
\hline MGPS & 10 & 4 & 6 & 7 \\
\hline
\end{tabular}

\subsection{Application of the AMEC multigas passive sampling system}

The MGPS has been used in many projects across Canada and in the world indoors and outdoors. The Palliser Airshed Society (PAS) project is an example. The PAS is located at southern Alberta, Canada (see Figure 1). There are many human being activities in the area including agriculture, industry, transportation, and so on. Twenty two passive sampler stations were installed in the area to monitor $\mathrm{SO}_{2}, \mathrm{NO}_{2}$, and $\mathrm{O}_{3}$.

The AMEC multigas passive samplers have been used in the airshed for almost 4 years. Very reasonable results have been obtained. Figure 9 shows the $\mathrm{SO}_{2}, \mathrm{NO}_{2}$, and $\mathrm{O}_{3}$ concentration distribution trends in the PAS.

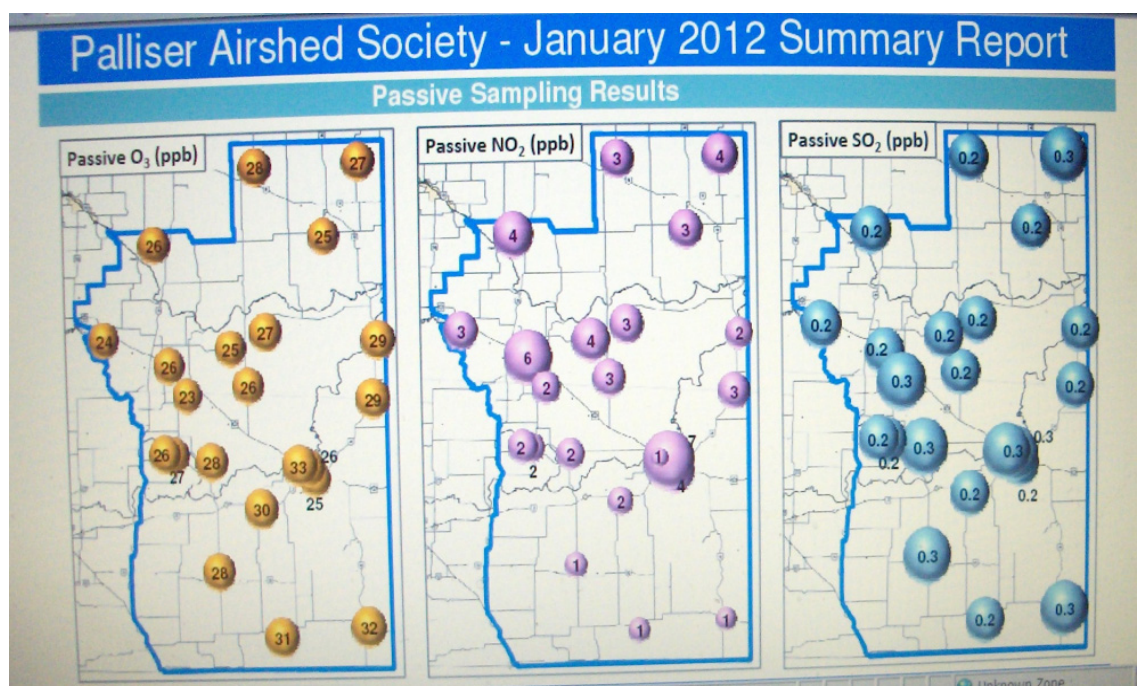

Figure 9: $\quad$ AMEC passive stations in the PAS.

This project also demonstrates the cost effective feature of MGPS (Table 3). Compared to ASPS, MGPS saves about $70 \%$ of major costs. 
Table 3: $\quad$ Comparison of ASPS and MGPS.

\begin{tabular}{|c|c|c|c|}
\hline ITEM & ASPS & MGPS & SAVE \% \\
\hline Pass body & 66 & 22 & 64 \\
\hline Diffusion barrier & 66 & 22 & 64 \\
\hline Collection filter & 66 & 22 & 75 \\
\hline Field job & 66 & 22 & 64 \\
\hline Waste generated & 66 & 22 & 75 \\
\hline
\end{tabular}

\section{Conclusion}

The newly patented passive sampling technology - AMEC multigas passive sampling system (MGPS) has played and will continuously play a very important role in the air pollution management, environment impact assessment and other air monitoring programs, especially in the Integrative Air Quality Management (Air monitoring zone) used in Canada, which is a cost effective way to address regional air quality and air pollution impact issues. The air monitoring zone can extendedly involve all stakeholders in the region and take care of every party's concerns.

\section{References}

[1] West central Airshed Society; www.wcas.com

[2] Clean Air Strategic Alliance; www.casadata.org

[3] Parkland airshed management zone; www.pamz.org

[4] Tang, H.; Brassard, B.; Brasssard R.; Peake, E.; A new passive sampling system for monitoring $\mathrm{SO} 2$ in the atmosphere, FACT, 1, pp. 307-315, 1997.

[5] Tang, H.; Lau, T.; Brassard B.; Cool, W., A new all-season passive sampling system for monitoring $\mathrm{NO}_{2}$ in air, FACT, 6, 338 - 345.

[6] Tang, H. and Lau, L., A new all season passive sampling system for monitoring ozone in air, Environ. Monit. Assess. 65, 129-137. 1999.

[7] Tang, H.; Sandeluk, J,; Lin L,; and Lown W.; A new all-season passive sampling system for sampling H2S in air, The ScientificWorld, 2, pp. 155168. 2002.

[8] Reid, P.; The role of passive ambient monitoring for sulphur dioxide in the northern Rocky Mountain foothills, in the proceedings of CPANS conference, Edmonton, AB, Canada, 2001.

[9] Tang, H. Paper in preparation, 2012.

[10] Higuchi, K.; Schaeffer, D.R.; Hirano, K.; Advanced monitoring method for air environment by Ogawa Passive, in Proceedings of Passive sampling workshop and symposium, Reston, VA, April 2007.

[11] Tang, H. and Apon, F. (2008) Multigas Passive Sampling System Tested, Canadian Environmental Protection, May-June 2008, pp. 20-21. 
528 Environmental Impact

[12] Hongmao Tang, Leon Burns and Fred Apon, (2011)AMEC Multigas Passive Sampling System - A Green Product for Cost-Effectively Monitoring Air Pollution Indoors and Outdoors, $19^{\text {th }}$ International Air Pollution conference, Multa, Sep19 - 212011.

[13] Palliser Airshed Society; www.palliserairshed.org 